\title{
Natural Implementation of Neutralino Dark Matter
}

\author{
S. F. King ${ }^{a, b 1}$, and J. P. Roberts ${ }^{b 2}$ \\ ${ }^{a}$ TH Division,Physics Department, \\ CERN, 1211,Geneva 23, Switzerland \\ ${ }^{b}$ School of Physics and Astronomy, University of Southampton, \\ Southampton, SO17 1BJ, U.K.
}

\begin{abstract}
The prediction of neutralino dark matter is generally regarded as one of the successes of the Minimal Supersymmetric Standard Model (MSSM). However the successful regions of parameter space allowed by WMAP and collider constraints are quite restricted. We discuss fine-tuning with respect to both dark matter and Electroweak Symmetry Breaking (EWSB) and explore regions of MSSM parameter space with non-universal gaugino and third family scalar masses in which neutralino dark matter may be implemented naturally. In particular allowing non-universal gauginos opens up the bulk region that allows Bino annihilation via t-channel slepton exchange, leading to "supernatural dark matter" corresponding to no fine-tuning at all with respect to dark matter. By contrast we find that the recently proposed "well tempered neutralino" regions involve substantial fine-tuning of MSSM parameters in order to satisfy the dark matter constraints, although the fine tuning may be ameliorated if several annihilation channels act simultaneously. Although we have identified regions of "supernatural dark matter" in which there is no fine tuning to achieve successful dark matter, the usual MSSM fine tuning to achieve EWSB always remains.
\end{abstract}

\footnotetext{
${ }^{1}$ E-mail: sfk@hep.phys.soton.ac.uk

${ }^{2}$ E-mail: jpr@phys.soton.ac.uk
} 


\section{Introduction}

One of the main arguments in favour of TeV scale Supersymmetry (SUSY) is that it provides a natural dark matter candidate [1]. In the minimal supersymmetric standard model (MSSM) with conserved R-parity the lightest supersymmetric particle (LSP) is absolutely stable. If the LSP is the lightest neutralino it is also neutral, weakly interacting and has a mass of order the electroweak scale. Although general arguments suggest that such a particle should provide a good dark matter candidate [2], the successful regions of parameter space allowed by WMAP and collider constraints are quite restricted [3]-32] and it is far from clear if the neutralino can be considered a natural dark matter candidate.

For example in the constrained MSSM (CMSSM) [3] the majority of the parameter space results in a value of $\Omega_{C D M} h^{2}$ that exceeds the observed value by orders of magnitude. This is not to say that the CMSSM is ruled out by the WMAP measurement, just that it requires some very precise relations between parameters to fit the theory to the observed data. In the CMSSM the only region in which we can fit all experimental limits within $2 \sigma^{1}$ is the $\tilde{\tau}$-coannihilation channel. In this region $m_{\tilde{\tau}} \approx m_{\tilde{\chi}_{1}^{0}}$ and the annihilation of SUSY particles in the early universe becomes extremely efficient. This effect is so large that the calculated value of $\Omega_{C D M} h^{2}$ drops by a factor of 100 as $m_{\tilde{\tau}}$ approaches $m_{\tilde{\chi}_{1}^{0}}$. At one point on this steep gradient of decreasing $\Omega_{C D M} h^{2}$, the model gives the right amount of dark matter. However to be within this strip the low energy masses must be tuned to within a few percent. From a low energy point of view the MSSM contains no justification for the stau and neutralino masses to fall within this region and this has led to claims 34] that such regions involve fine-tuning. Instead Arkani-Hamed, Delgado and Giudice argue that "Well Tempered Neutralino" regions in which the neutralino is not pure Bino but a Bino/Higgsino or Bino/Wino mix are more plausible.

The present paper is concerned with the question of how to implement neutralino dark matter in a natural way within the general MSSM. Specifically we explore regions of MSSM parameter space with non-universal gaugino and third family scalar masses in which neutralino dark matter may be implemented naturally. Dark matter with nonuniversal third family scalar masses has been considered in [17] motivated by purely phenomenological consideration, or in [18, 20] motivated by specific GUT models. The collider phenomenology of such models has also been studied in [19]. Dark Matter with non-universal gaugino masses has also been studied in [4]-[10] and particular high energy models with non-universal gauginos have been analysed in [11]-[16, [20]. In both cases the question of naturalness of dark matter has not been addressed. Moreover no analysis of any kind has been performed which considers both types of non-universality together. Here we extend the above analyses considerably by studying the question of both dark matter and electroweak fine-tuning for both non-universal third family

\footnotetext{
${ }^{1}$ This statement is true if one takes seriously the discrepancy between the reported value of the anomalous magnetic moment $g-2$ of the muon. Throughout this paper we take the measured value of $\delta a_{\mu}$ that provides a $2.7 \sigma$ deviation from the Standard Model [33. If this discrepancy were ignored, it would be possible to access the focus point and Higgs funnel regions of the CMSSM parameter space. However these turn out to be finely tuned regions as we shall discuss later.
} 
scalar masses and non-universal gauginos, including a first study of the effect of both types of non-universality together.

Our main focus in this paper is on the question of whether and how naturalness may be improved by allowing such types of non-universality. In order to examine the relative naturalness of different regions we employ a dark matter fine-tuning sensitivity parameter, which we use in conjunction with the similarly defined sensitivity parameter used for electroweak symmetry breaking (EWSB) [35], 42. Employing these quantitative measures of fine-tuning we find that $\tilde{\tau}$-coannihilation channel in the CMSSM may involve as little as $25 \%$ tuning, due to renormalisation group (RG) running effects. This result is in agreement with [51]. In general moving beyond the framework of universal soft sfermion and gaugino masses in the CMSSM the structure of neutralino annihilation changes drastically. By allowing third family scalar masses to vary independently [17, [18, we can access regions in which the LSP has a significant Higgsino fraction as well as regions in which the dominant annihilation is through coannihilation with selectrons and smuons. With non-universal soft gaugino masses [4] we can have a well tempered neutralino that is a Bino/Wino mix, although this is also very fine-tuned. However allowing non-universal gauginos also opens up a favourable region (excluded in the CMSSM) with light sleptons that allows Bino annihilation via t-channel slepton exchange. This yields a bulk region of parameter space leading to "supernatural dark matter" where successful dark matter can be achieved with no fine-tuning at all, leaving only the usual fine-tuning required to achieve electroweak symmetry breaking.

The paper is set out as follows. Our methodology is stated in section 2. In section 3 we survey the physical bounds that constrain our parameter space, especially $B R(b \rightarrow$ $s \gamma), \delta a_{\mu}$ and $\Omega_{C D M} h^{2}$, we discuss radiative electroweak symmetry breaking, and we define the dark matter and EWSB sensitivity parameters that provide a measure of fine-tuning. In section 4 , to set the scene, we consider the coannihilation region in the CMSSM, which provides us with a useful reference point against which the subsequent non-universal cases may be compared. In section [5 we allow the third family soft sfermion mass squared to vary independently. In section [ w we consider neutralino dark matter with non-universal gaugino masses, but with a universal soft scalar mass. In section 7 we consider both the effects of including an independent third family sfermion mass squared and non-universal soft gaugino masses. Section 8 concludes the paper.

\section{Methodology}

As the inputs for models such as the CMSSM are defined at $M_{G U T} \approx 2 \times 10^{16} \mathrm{GeV}$ we need to use the RGEs to produce a mass spectrum at the electroweak breaking scale. To do this we use SOFTSUSY v.1.9.1[55]. Once we have generated the low energy spectrum for a point we pass it to micrOMEGAs v.1.3.6 [56] to calculate $\Omega_{C D M} h^{2}, \delta a_{\mu}$ and $B R(b \rightarrow s \gamma)$. We reject points that do not provide REWSB, that violate particle search limits from LEP2, points that produce a tachyon and any points that produce an LSP that is not the neutralino. In the remaining parameter space we plot the 1 and $2 \sigma$ bounds of $\Omega_{C D M} h^{2}, \delta a_{\mu}$ and $B R(b \rightarrow s \gamma)$. Throughout we take the top mass to be 


\section{Physical Bounds}

\section{$3.1 \quad B R(b \rightarrow s \gamma)$}

The variation of $B R(b \rightarrow s \gamma)$ from the value predicted by the Standard Model is highly sensitive to SUSY contributions. To date no variation from the Standard Model has been detected. The present experimental measurement comes from BELLE [57], CLEO [58] and ALEPH [59. We follow the analysis of [17] and take the value to be:

$$
B R(b \rightarrow s \gamma)=(3.25 \pm 0.54) \times 10^{-4}
$$

The 1-loop SUSY processes involve loops with a charged Higgs and a top quark and loops with a chargino and a squark. Though the full 1-loop calculation is complicated, here we only mention that we expect contributions to be enhanced whenever the intermediate particles are light. There is also an enhancement for large $\tan \beta$.

Within micrOMEGAs, all 1-loop effects are included and some 2-loop contributions. There is a detailed discussion of their implementation in [56]. We do not include theoretical errors in our analysis as they are hard to estimate at this stage.

\subsection{Muon $g-2$}

Present measurements of the value of the anomalous magnetic moment of the muon $a_{\mu}$ deviate from the theoretical calculation of the SM value. However questions remain around the exact form of the standard model calculation, specifically whether we should use $\tau$ or $e^{+} e^{-}$data in the analysis of the hadronic vacuum polarisation. At ICHEP '04 60] there was general agreement that the $\tau$ data. Here we take the result obtained by using the $e^{+} e^{-}$data and consider its implications for a SUSY theory. With the present experimental value from [33] and the theoretical calculation of the SM value from [61] there is a discrepancy:

$$
\left(a_{\mu}\right)_{\exp }-\left(a_{\mu}\right)_{S M}=\delta a_{\mu}=(2.52 \pm 0.92) \times 10^{-9}
$$

This amounts to a $2.7 \sigma$ deviation from the standard model. ${ }^{2}$

The SUSY contributions to $a_{\mu}$ come from penguin diagrams of two types. One is mediated by a chargino and a muon sneutrino, the other is mediated by a neutralino and

\footnotetext{
${ }^{2}$ Note that the SND Collaboration recently reported a result that was out of line with the $e^{+} e^{-}$ results from other groups, being more consistent with the $\tau$ data. However very recently an error was reported in their analysis, and now the most recent result from SND is completely consistent with the $e^{+} e^{-}$results from other groups 62 . This effectively will serve to increase the discrepancy of the muon $g-2$ with the standard model beyond $2.7 \sigma$, but since the new analysis has not yet been performed here we shall continue to conservatively assume the $2.7 \sigma$ deviation.
} 
a smuon. For a detailed discussion of these contributions, see [63. For our purposes it is enough to note that there will be enhancements to the SUSY contribution whenever smuons, mu-sneutrinos, charginos and neutralinos become light.

\section{$3.3 \Omega_{C D M} h^{2}$}

Evidence from the CMB and rotation curves of galaxies both point to a large amount of cold non-baryonic dark matter in the universe. The present measurements 64] place the dark matter density at:

$$
\Omega_{C D M} h^{2}=0.1126 \pm 0.0081
$$

Due to R-parity, the lightest supersymmetric particle (LSP) is stable. If it is also electrically neutral, weakly interacting and massive it is a prime candidate for dark matter. In the majority of cases, just such a candidate exists in the neutralino. This is a mixture of the superpartners to $\mathrm{U}(1)$ and $\mathrm{SU}(2)$ gauge bosons of the standard model and the superpartners to the neutral Higgs bosons:

$$
\tilde{\chi}_{1}^{0}=N_{11} \tilde{B}+N_{12} \tilde{W}+N_{13} \tilde{H}_{1}^{0}+N_{14} \tilde{H}_{2}^{0}
$$

where $N_{1, j}$ are the relevant components of the matrix that diagonalises the low energy neutralino mass matrix.

This leads to three limiting cases:

$$
\begin{array}{ll}
\left|N_{11}\right|^{2} \approx 1 & \text { Bino dark matter } \\
\left|N_{12}\right|^{2} \approx 1 & \text { Wino dark matter } \\
\left|N_{13}\right|^{2}+\left|N_{14}\right|^{2} \approx 1 & \text { Higgsino dark matter }
\end{array}
$$

In many cases of pure Bino dark matter, t-channel slepton exchange is suppressed due to the sleptons being too heavy, resulting in a value of $\Omega_{C D M} h^{2}$ above the measured value. On the other hand pure Higgsino or Wino dark matter result in $\Omega_{C D M} h^{2}$ being too small. This leads to the plausible suggestion of the "well-tempered" neutralino [34] consisting of a roughly equal Bino/Wino or Bino/Higgsino mixed LSP. For example the well tempered Bino/Higgsino mixed LSP is achievable within the CMSSM in the so called Focus Point region where $m_{0}$ is large and $\mu$ is small 65. On the other hand the well tempered Bino/Wino mixed LSP is not achievable within the CMSSM, and requires non-universal gaugino masses.

Alternatively pure Bino dark matter is still viable providing annihilation channels are enhanced for some reason. For example in the CMSSM the so called Coannihilation region is viable, where the Bino LSP is close in mass to the stau slepton leading to similar abundances of Binos and staus in the early universe, allowing efficient coannihilation into $Z$ s and taus, for example, via t-channel neutralino exchange. Another example in the CMSSM is the so called Funnel region where $\tan \beta$ is large and the mass of the Bino LSP is equal to half the mass of the CP-odd pseudoscalar allowing efficient resonance annihilation. 
Both "well tempered" case and the enhanced Bino annihilation types of region are the exception rather than the rule. In the first case we need to tune the neutralino composition. In the second we need to tune the rest of the sparticle mass spectrum. The study of such tuning and how it may be overcome in certain regions of non-universal MSSM parameter space is the main subject of this paper.

\subsection{Radiative Electroweak Symmetry Breaking}

One fundamental requirement of any SUSY theory is that it include radiative electroweak symmetry breaking (REWSB). For a detailed review of this process, see [1]. Here we merely summarise the consequences of imposing REWSB. Firstly, by requiring REWSB, we swap the soft SUSY breaking parameters $\mu$ and $b$ for $\tan \beta$ and $\operatorname{sign}(\mu)$. The size of $\mu^{2}$ is determined by:

$$
\mu^{2}(t)=\frac{m_{H_{d}}^{2}(t)-m_{H_{u}}^{2}(t) \tan ^{2} \beta}{\tan ^{2} \beta-1}-\frac{1}{2} m_{Z}^{2}(t)
$$

where $t=\log Q$ and $Q$ is the energy scale at which we want to determine $\mu$. For REWSB to exist in a given model, we must have a positive value of $\mu^{2}$ at the low energy scale $Q \sim m_{Z}$.

$\tan \beta$ and $m_{Z}$ are easily determined at the low energy scale. $\tan \beta$ is considered as a free input for all cases considered and so just takes the value chosen for the model point under consideration. $m_{Z}$ is just the experimentally measured running mass of the $Z^{0}$. All the dependence of $\mu^{2}$ on the soft parameters is tied up in the $m_{H_{u}, H_{d}}^{2}$ terms. These masses must be evaluated at the low energy scale and so we must consider the RGE evolution from the soft scale to understand how varying the soft masses will affect $\mu^{2}$.

It is worth noting that the $m_{H_{d}}^{2}$ term in Eq. 4 is suppressed relative to the $m_{H_{u}}^{2}$ term by a factor of $1 / \tan ^{2} \beta$. Therefore, for all but very small values of $\tan \beta$, the size of $\mu^{2}$ will be dominated by $m_{H_{u}}^{2}$. For large $\tan \beta$ (say larger than 2) Eq. 4 simplifies to:

$$
\mu^{2}(t) \approx-m_{H_{u}}^{2}(t)-\frac{m_{Z}^{2}(t)}{2}
$$

To obtain a positive value of $\mu^{2}$ in this limit we clearly need a negative value of $m_{H_{u}}^{2}$ at the low energy scale. As we have positive values for $m_{H_{u}, d}^{2}$ at $M_{G U T}$ in the models we consider, we must force $m_{H_{u}}^{2}$ negative through running effects.

It is possible to calculate $\mu^{2}$ explicitly as a function of the high energy soft parameters 42] (here we take $\tan \beta=10$ as we consider this region extensively throughout this work): 


$$
\begin{aligned}
\frac{m_{Z}^{2}}{2}=\quad & -0.94 \mu^{2}+0.010 m_{H_{1}}^{2}-0.19 M_{2}^{2}-0.0017 M_{1}^{2}-0.63 m_{H_{2}}^{2}+0.38 m_{Q_{3}}^{2} \\
& +0.38 m_{U_{3}}^{2}+0.093 A_{t}^{2}-0.011 A_{t} M_{1}-0.070 A_{t} M_{2}-0.30 A_{t} M_{3} \\
& +2.51 M_{3}^{2}+0.0059 M_{1} M_{2}+0.028 M_{1} M_{3}+0.195 M_{2} M_{3}
\end{aligned}
$$

This formula gives the condition amongst the high energy soft masses to achieve correct REWSB. It clearly shows that fine tuning is necessary if soft masses greatly exceed $m_{Z}$ (as they must). Correct REWSB clearly requires that the negative terms must not be too large compared to the positive terms, for example $m_{H_{2}}^{2}$ and $M_{2}^{2}$ must not be to large compared to $m_{Q_{3}}^{2}$ and $M_{3}^{2}$. Along the edge of regions where we fail to achieve REWSB, we will have regions with small $\mu$. These are favourable for two reasons. Firstly, such regions will facilitate neutralino annihilation via t-channel chargino exchange. Secondly, small $\mu$ means small fine-tuning of the $\mu$ parameter.

\subsection{Fine-tuning sensitivity parameters}

The naturalness of radiative electroweak symmetry breaking from supersymmetry has been extensively studied [35]-[49]. In such studies, the following measure is commonly used to quantify the degree of fine-tuning required for a given model point:

$$
\Delta_{a}^{\mathrm{EW}}=\frac{\partial \ln \left(m_{Z}^{2}\right)}{\partial \ln (a)}
$$

where $a$ includes all the soft parameters $m_{\text {soft }}$ at the GUT scale together with $\tan \beta$. In this paper we wish to study the naturalness of dark matter. To this end we use an analagous measure to quantify the degree of fine-tuning required to reproduce the observed dark matter density:

$$
\Delta_{a}^{\Omega}=\frac{\partial \ln \left(\Omega_{C D M} h^{2}\right)}{\partial \ln (a)}
$$

Though the measures are analagous, the calculations of radiative electroweak symmetry breaking and the calculation of the relic density of dark matter have important differences. In the case of electroweak symmetry breaking, the soft masses that define SUSY breaking provide a complete set of inputs. This is not the case for dark matter. The calculation of the present day relic density necessarily involves some assumptions about the cosmology of the early universe. These assumptions are:

- At some point in the universe's history (after inflation) there was a radiation dominated period in which $T \gg m_{\chi}$, where $m_{\chi}$ is the mass of the LSP.

- There are no exotic non-thermal production methods for dark matter. 
If the first assumption holds then there was a period in the history of the universe in which standard model matter and supersymmetric matter (particularly the LSP) were in equilibrium. As the universe expands and cools a relic of stable SUSY particles is left behind. No further assumptions are required in the calculation of this relic density.

If the second assumption holds then this LSP relic density is the only dark matter present in the universe. In this paper we work within such a cosmological framework. Within such a framework, the soft masses that parameterise the structure of SUSY breaking also uniquely define the relic density of dark matter. As a result, we can use Eq 8 to quantify the naturalness of dark matter. In more exotic cosmologies, the set of input parameters $a$ would need to be expanded to include variations in the details of early universe cosmology but such cosmologies are beyond the scope of this paper.

A similar measure to $\mathrm{Eq} 8$ was previously defined and used to study the CMSSM in [50, 51]. One motivation of these studies was to study the sensitivity that the LHC would need to achieve to give a corresponding accuracy in the predicted dark matter density. This is an interesting question and our results can also be applied in this context, though we do not focus on that here. In contrast to [51, we define the fine tuning of a model point to be equal to the largest individual tuning ${ }^{3}: \Delta^{\Omega}=\max \left(\Delta_{a}^{\Omega}\right)$.

Note that the above sensitivity parameters in the electroweak sector have been criticized in 552-54 where an alternative measure of fine-tuning was proposed that arguably gives a more reliable estimate of fine-tuning. Essentially the authors of [52][54] point out that such sensitivity parameters do not provide a reliable measure of fine-tuning unless they are normalized to some average value of sensitivity for that particular parameter. Thus they advocate using instead the fine-tuning parameter $\gamma_{a}$ defined by,

$$
\gamma_{a}=\frac{\Delta_{a}}{\bar{\Delta}_{a}}
$$

where the average value $\bar{\Delta}_{a}$ is defined as discussed in [52]-[54]. This definition in Eq9 gives a sense to the numerical value of the sensitivity parameter, for example it may be the case that all values of the sensitivity parameter are large over the entire theoretically allowed range of input parameters. Fine tuning then corresponds to some unusually high levels of sensitivity above the typical values, where the typical values are themselves high.

In the present paper it is sufficient to consider the unnormalized sensitivity parameters $\Delta_{a}$ for dark matter. For dark matter we shall find regions of parameter space where the sensitivities are very low, indeed sometimes less than unity. Conversely we shall find other regions of parameter space where the sensitivity parameters are very high, sometimes as high as of order $10^{3}$. The existence of regions of parameter space with low sensitivities shows that the issue of the normalization of the sensitivity parameters is not so crucial in the case of dark matter. Therefore we shall only calculate the absolute values of the sensitivity parameters which is sufficient to compare the relative sensitivies of different regions of parameter space.

\footnotetext{
${ }^{3}$ There is a divide in the literature as to whether $\Delta=\max \left(\Delta_{a}\right)$ or $\Delta=\sqrt{\sum_{a} \Delta_{a}^{2}}$. Which definition one takes to be more accurate is a matter of taste. Throughout the paper we take $\Delta=\max \left(\Delta_{a}\right)$
} 


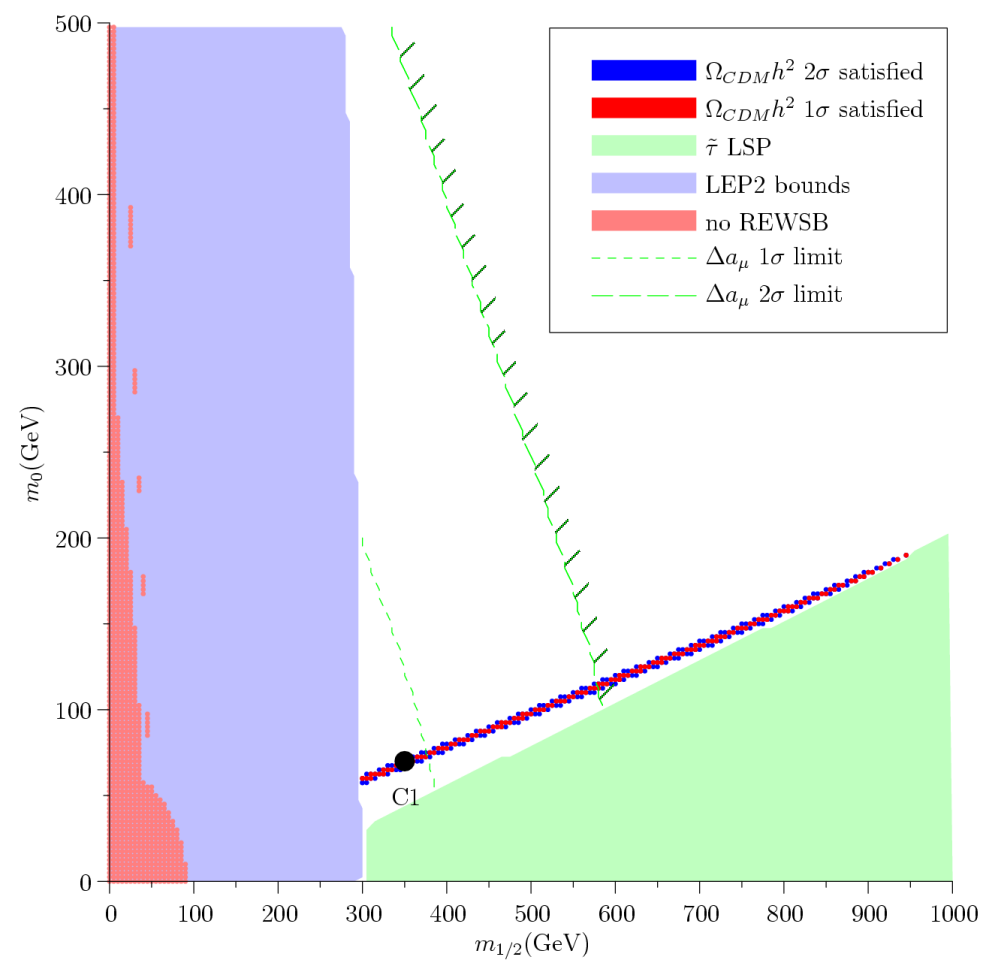

Figure 1: The $\left(m_{1 / 2}, m_{0}\right)$ plane for the CMSSM with $A_{0}=0, \tan \beta=10$.

\section{CMSSM}

The simplest model of SUSY breaking is the CMSSM ${ }^{4}$. In such a model all soft mass matrices are diagonal at the high scale, taken here to be $M_{G U T}$, and have a universal mass squared, $m_{0}^{2}$, between generations. The gauginos also have a universal soft mass $m_{1 / 2}$. After imposing REWSB we have 4 free parameters and a sign:

$$
m_{0}, m_{1 / 2}, \tan \beta, A_{0} \text { and } \operatorname{sign}(\mu)
$$

Studies of the CMSSM find three regions that satisfy dark matter bounds. At high $m_{0}, \mu$ can become very small which in turn results in a Higgsino/Bino neutralino - the Focus Point region. At large $\tan \beta(\approx 50)$ and moderately large $m_{1 / 2}$ the pseudoscalar Higgs boson can become quite light giving rise to region in which $2 m_{\tilde{\chi}_{1}^{0}} \approx m_{A}$. Here neutralino annihilation proceeds through s-channel exchange of a pseudoscalar Higgs boson, known as the Higgs Funnel. The third region that reproduces the observed value of $\Omega_{C D M} h^{2}$ is where we have coannihilation of the neutralino with the right handed stau. This region appears at low values of $m_{0}$ and $m_{1 / 2}$ where $m_{\tilde{\tau}} \approx m_{\tilde{\chi}_{1}^{0}}$. This region has a light SUSY spectrum and satisfies $\delta a_{\mu}$ and $B R(b \rightarrow s \gamma)$ at $1 \sigma$.

In Fig. 1 we study the CMSSM in the $\left(m_{1 / 2}, m_{0}\right)$ plane with $A_{0}=0, \tan \beta=10$ and $\operatorname{sign}(\mu)$ positive. Low $m_{1 / 2}$ values are excluded by LEP2 bounds on the lightest

\footnotetext{
${ }^{4}$ See [3] for a comprehensive discussion of the CMSSM parameter space. Here we merely present the most favourable region for the purpose of comparison with our later results.
} 
Higgs mass. Low $m_{0}$ values are excluded as they result in the stau becoming the LSP. In the remaining parameter space we plot the 1 and $2 \sigma$ bounds on $\delta a_{\mu}$ and $\Omega_{C D M} h^{2}$. $B R(b \rightarrow s \gamma)$ is satisfied at $1 \sigma$ through the entire parameter space and so does not appear as a bound. We have considered relatively low values of $m_{0}$ and $m_{1 / 2}$ as it is only in these regions that the CMSSM can satisfy $\delta a_{\mu}$.

The region in which the CMSSM reproduces the observed value of $\Omega_{C D M} h^{2}$ is shown as a thin strip containing the point C1 running close the stau LSP light shaded region (the coannihilation strip is coloured red and blue for the $1 \sigma$ and $2 \sigma$ regions respectively). The coannihilation strip is the only viable dark matter region for the range of parameters in Fig. 1. The thinness of the stau coannihilation strip indicates that some degree of tuning is required. Before we go on to study how natural the region is, first we need to consider the neutralino annihilation channels that come into play in the calculation of the relic density.

Through all the displayed parameter space the lightest neutralino is the Bino. Bino dark matter can always annihilate through the process $\tilde{\chi}_{1}^{0} \tilde{\chi}_{1}^{0} \rightarrow f \bar{f}$ via t and u-channel sfermion exchange where $f, \bar{f}$ are standard model fermions. This process becomes less efficient as the mass of the intermediate sfermions increase. However as low $m_{0}$ and $m_{1 / 2}$ are excluded, the sfermions of the CMSSM are never light enough for $\tilde{\chi}_{1}^{0} \tilde{\chi}_{1}^{0} \rightarrow f \bar{f}$ to account for the observed relic density. $\Omega_{C D M} h^{2}=\mathcal{O}(1)$ across the majority of the parameter space.

To avoid overclosing the universe with an overabundance of dark matter we need another annihilation channel. This happens when $m_{\tilde{\tau}_{R}} \approx m_{\tilde{\chi}_{1}^{0}}$. In this case there is a significant number density of staus at the time of freeze out. This has two effects. Firstly, we must include processes of the form $\tilde{\tau} \tilde{\tau} \rightarrow$ SM particles and $\tilde{\tau} \tilde{\chi}_{1}^{0} \rightarrow$ SM particles in our calculation of the relic density of neutralinos. Secondly, it means that interactions will continue to occur long after neutralinos on their own would have frozen out $^{5}$. The combination of effects results in a substantial decrease in the relic density. Unfortunately this generally leads to $\Omega_{C D M} h^{2}=\mathcal{O}(0.01)$. Nevertheless there is a band in which there is just enough coannihilation. This is just the coannihilation strip in Fig. 1 that lies just above the region in which the $\tilde{\tau}$ is the LSP.

As we move from one side of the strip to the other $\Omega_{C D M} h^{2}$ varies by roughly 2 orders of magnitude. Coannihilation will only provide the right amount of dark matter today if $m_{\tilde{\tau}}\left(m_{Z}\right)$ and $m_{\tilde{\chi}_{1}^{0}}\left(m_{Z}\right)$ are correlated to a precision of a few percent. In [34] Arkani-Hamed, Delgado and Giudice use this critical sensitivity to the masses to claim that coannihilation requires fine-tuning. However we can only talk about fine-tuning in the context of a high energy model whereas their consideration of coannihilation is to consider the sensitivity on the low energy masses. To quantify the degree of fine-tuning in the coannihilation region, we take the point $\mathrm{C} 1$ with $m_{0}=70 \mathrm{GeV}, m_{1 / 2}=350 \mathrm{GeV}$, $\tan \beta=10, A_{0}=0$ and allow each input parameter to vary individually. Using the measure defined in Eq. 8 we can quantify the sensitivity of the coannihilation strip to variations in each soft parameter at $m_{G U T}$, and this is done in Table 1].

\footnotetext{
${ }^{5}$ For a detailed study of coannihilation in general and the CMSSM specifically see [66] and [67] respectively.
} 


\begin{tabular}{|l|l|}
\hline Parameter & Value \\
\hline$\Delta_{m_{0}}^{\Omega}$ & 3.5 \\
$\Delta_{m_{1 / 2}}^{\Omega}$ & 3.4 \\
$\Delta_{\tan \beta}^{\Omega}$ & 1.4 \\
$\Delta_{A_{0}}^{\Omega}$ & 0 \\
\hline$\Delta^{\Omega}$ & 3.5 \\
\hline$\Delta^{\mathrm{EW}}$ & 160 \\
\hline
\end{tabular}

Table 1: Fine tuning sensitivity parameters in the CMSSM with $A_{0}=0, \tan \beta=10$ at point $\mathrm{C} 1$ in Fig. 1 with $m_{0}=70 \mathrm{GeV}, m_{1 / 2}=350 \mathrm{GeV}$.

As coannihilation depends on the mass difference between the stau and the neutralino, we would expect the calculation of $\Omega_{C D M} h^{2}$ to be sensitive to $m_{0}$ and $m_{1 / 2}$. However the sensitivity of $\Omega_{C D M} h^{2}$ to these parameters is only around $25 \%$, considerably less than would be expected from considerations of the low energy masses alone. This is because along the coannihilation strip $m_{0}<m_{1 / 2}$ and so the masses of both the sleptons and the neutralino are primarily dependent on the same parameter $m_{1 / 2}$, in the case of the stau through RG running effects.

Though here we only study coannihilation, there are other regions of the CMSSM parameter space in which we can reproduce the observed relic density, namely the Focus Point and Higgs Funnel regions. These appear at large $m_{0}$ and $\tan \beta$ respectively and do not allow agreement with $\delta a_{\mu}$. However it is worth briefly mentioning that under the fine-tuning measure $\Delta^{\Omega}$, the Focus Point region with Bino/Higgsino dark matter has $\Delta^{\Omega} \approx 60$ and the Higgs Funnel region has $\Delta^{\Omega} \approx 30$. Thus in the CMSSM, coannihilation provides more natural dark matter than the well-tempered neutralino of the Focus Point region.

However this region does still exhibit fine-tuning. We now go on to consider models beyond the CMSSM to look for natural dark matter. We take point C1 as a datum to which we will compare regions that satisfy $\Omega_{C D M} h^{2}$ once we introduce varying degrees of non-universality in our soft parameters.

\section{Non-universal Third Family Scalar Masses}

The first deviation we take from universality of the CMSSM is to allow the 3rd family sfermion mass squared to vary independently of the 1st and 2 nd families. This results in a model with five parameters and a sign:

$$
m_{0}, m_{0,3}, m_{1 / 2}, \tan \beta, A_{0} \text { and } \operatorname{sign}(\mu)
$$

These determine the soft masses of the squarks, sleptons, Higgs and gauginos at $M_{G U T}$ to be: 


$$
\begin{aligned}
m_{\tilde{Q}}^{2}, m_{\tilde{L}}^{2}, m_{\tilde{u}}^{2}, m_{\tilde{d}}^{2}, m_{\tilde{e}}^{2} & =\left(\begin{array}{ccc}
m_{0}^{2} & 0 & 0 \\
0 & m_{0}^{2} & 0 \\
0 & 0 & m_{0,3}^{2}
\end{array}\right) \\
m_{H_{u}}^{2}=m_{H_{d}}^{2} & =m_{0,3}^{2} \\
M_{\alpha} & =m_{1 / 2}
\end{aligned}
$$

where $\alpha=1,2,3$ labels the three gauginos. We have set the Higgs soft masses to be equal to the third family soft mass $m_{0,3}$ since it seems reasonable that all soft masses involved in EWSB should be of the same order. Also this is the case in certain string models of non-universal third family scalar masses [68, 69.

From a purely phenomenological point of view, we gain a lot by allowing ourselves this extra freedom as pointed out in [17]. Firstly, the size of $\mu$ is primarily sensitive to the third family squark masses and the Higgs masses as shown in Eq. 6. On the other hand $\delta a_{\mu}$ is sensitive to the first and second family slepton masses. If we allow the 3rd generation soft masses to vary independently of the 1 st and 2 nd, we can access regions with low $\mu$ in which the neutralino is a Bino/Higgsino mix and still agree with $\delta a_{\mu}$ at $1 \sigma$.

However we have to be careful with low values of $\mu$. Small $\mu$ results in light charginos which enhance the SUSY contribution to $B R(b \rightarrow s \gamma)$. However the charginos appear in loops with stops, and with a large value of $m_{0,3}$, the stops become heavy and help to suppress the contribution. Thus in this model, $B R(b \rightarrow s \gamma)$ will be problem at large $m_{0,3}$ but not as much as might be initially expected.

In Fig. 2 we take the same range for $m_{0}$ and $m_{1 / 2}$ as we did for Fig. 4 but we now set $m_{0,3}=1000 \mathrm{GeV}$. With a large value of $m_{0,3}$ the stau is no longer the lightest slepton, we now have a normal mass hierarchy $(\mathrm{NMH})$ in the sfermions. The immediate result of this is that the coannihilation strip at low $m_{0}$ is now coannihilation with selectrons and smuons rather than staus. This also means that we can now access $m_{0}=0$ without ending up with a charged LSP. This happens at $m_{1 / 2}=230 \mathrm{GeV}$. The lower bound on $m_{1 / 2}$ from LEP2 constraints on the lightest Higgs also changes between Fig. 1 and Fig. 2 from $300 \mathrm{GeV}$ to $m_{1 / 2} \approx 200 \mathrm{GeV}$.

As we can access low values of both $m_{1 / 2}$ and $m_{0}$, we can access regions with light sleptons that in turn mediate the process $\tilde{\chi}_{1}^{0} \tilde{\chi}_{1}^{0} \rightarrow f \bar{f}$. As we move to low $m_{0}$ and $m_{1 / 2}$ this process becomes competitive with coannihilation. This shows up as a widening of the coannihilation region at low $m_{0}$ and $m_{1 / 2}$. However accessing this region comes at a cost. For low $m_{0}$ and $m_{1 / 2}<250 \mathrm{GeV}$ the smuons become so light that the SUSY contribution to $\delta a_{\mu}$ is too large. This large contribution could be reduced if we were to allow $M_{1}$ and $M_{2}$ to have different signs. Finally the dashed red line is also an early warning. For $m_{1 / 2}<350 \mathrm{GeV}$ the charginos are light enough that we violate the bounds on $B R(b \rightarrow s \gamma)$, though only at $1 \sigma$.

We consider two points on the successful dark matter strip: $\mathrm{S} 1$ at $m_{0}=50 \mathrm{GeV}$, $m_{1 / 2}=350 \mathrm{GeV}$; and $\mathrm{S} 2$ at $m_{0}=0 \mathrm{GeV}, m_{1 / 2}=230 \mathrm{GeV}$. The fine-tuning of these 


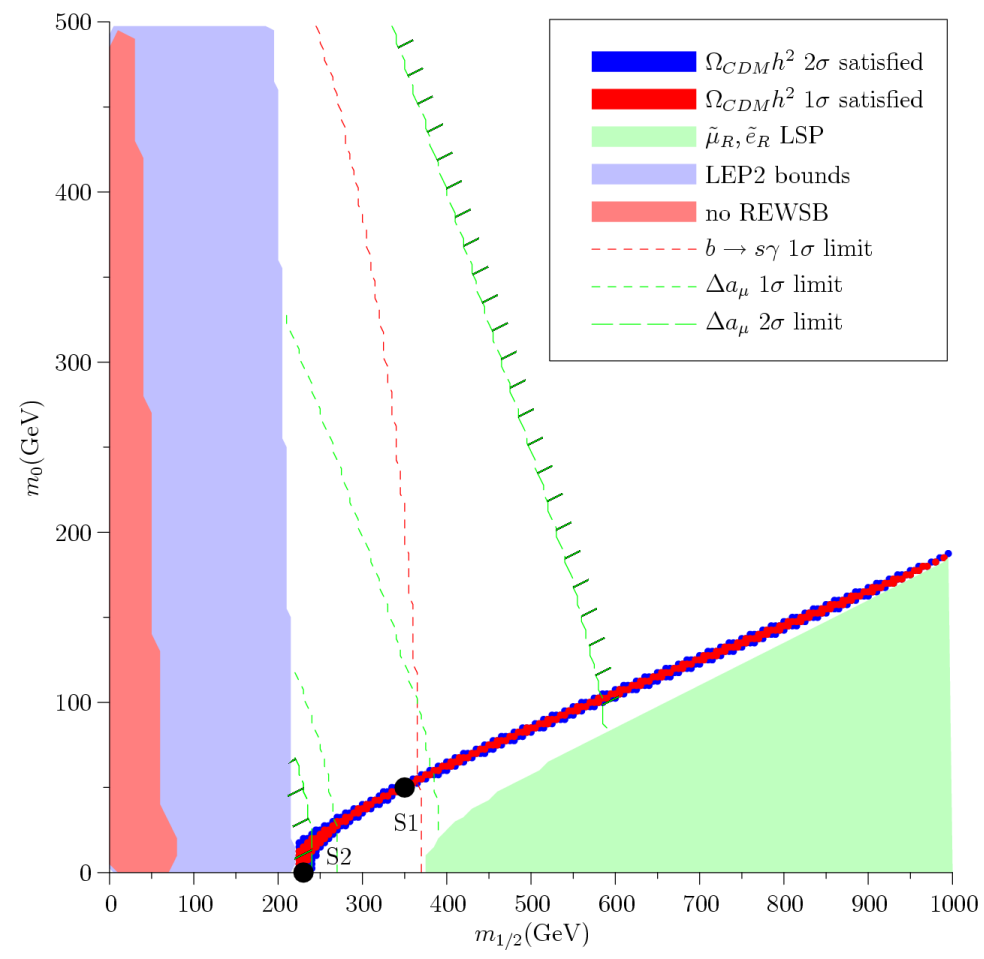

Figure 2: The $\left(m_{1 / 2}, m_{0}\right)$ plane for non-universal sfermion masses with $m_{0,3}=1 \mathrm{TeV}$, $A_{0}=0, \tan \beta=10$.

points with respect to our 5 parameters is shown in Table 2

We choose point S1 to allow direct comparison with the CMSSM. Once again we find a tuning of around $25 \%$ with respect to $m_{1 / 2}$ though the dependence on $m_{0,3}$ is minimal. This shouldn't be surprising as the neutralino annihilation channels in question depend primarily on the mass of the smuon, selectrons and the neutralino, none of which are sensitive to $m_{0,3}$. From this study, there is nothing to suggest that selectron and smuon coannihilation channels are any more natural that the stau coannihilation channel in the CMSSM.

Point S2 is considerably more interesting. Here there is a dramatic decrease in the sensitivity to the soft parameters. The primary reason for this is that there are more channels at work than just coannihilation. At $m_{0}=0$, the selectron and smuon are light enough that t-channel slepton exchange in the process $\tilde{\chi}_{1}^{0} \tilde{\chi}_{1}^{0} \rightarrow e^{+} e^{-}$or $\mu^{+}, \mu^{-}$becomes competitive. Indeed at this point such processes account for $60 \%$ of the annihilation of SUSY matter whereas coannihilation processes only account for $40 \%$.

This combination of annihilation channels is responsible for the drastic decrease in dark matter sensitivity to the soft parameters. By decreasing $m_{1 / 2}$ we decrease the mass of the neutralino and to lesser extent the selectron and the smuon. This increases the mass splitting between the states, suppressing coannihilation effects. However lower slepton masses enhance the cross-section for t-channel slepton exchange. This has the effect of smearing out the region of successful dark matter in the $m_{1 / 2}$ direction. 


\begin{tabular}{|l|l|l|}
\hline \multirow{2}{*}{ Parameter } & \multicolumn{2}{|l|}{ Value } \\
\cline { 2 - 3 } & $\mathrm{S} 1$ & $\mathrm{~S} 2$ \\
\hline$\Delta_{m_{0}}^{\Omega}$ & 2.4 & 0 \\
$\Delta_{m_{0,3}}^{\Omega}$ & 0.15 & 0.30 \\
$\Delta_{m_{1 / 2}}^{\Omega}$ & 4.2 & 1.8 \\
$\Delta_{\tan \beta}^{\Omega}$ & 0.061 & 0.033 \\
$\Delta_{A_{0}}^{\Omega}$ & 0 & 0 \\
\hline$\Delta^{\Omega}$ & 4.2 & 1.8 \\
\hline$\Delta^{\mathrm{EW}}$ & 240 & 200 \\
\hline
\end{tabular}

Table 2: Fine tuning sensitivity parameters for different points in Fig. 2 with $m_{0,3}=$ $1 \mathrm{TeV}, A_{0}=0, \tan \beta=10$. S1 has $m_{0}=50 \mathrm{GeV}, m_{1 / 2}=350 \mathrm{GeV}$. S2 has $m_{0}=0 \mathrm{GeV}, m_{1 / 2}=230 \mathrm{GeV}$

If $m_{0}=0, \Delta_{m_{0}}^{\Omega}=0$ automatically ${ }^{6}$. However we would also expect $\Delta_{m_{0}}^{\Omega}$ to be small whenever $m_{0}$ is small. Whenever $m_{0} \ll m_{1 / 2}$ the masses of both the sleptons and the neutralino are going to be primarily dependent upon $m_{1 / 2}$. Therefore the low energy phenomenology will be dominated by $m_{1 / 2}$ so neither t-channel slepton exchange or coannihilation should depend strongly on $m_{0}$ when $m_{0}$ is small.

This particular combination of channels combine to smear out critical dependence on the soft parameters. Moreover, both channels will naturally occur in any model with light $m_{0}$ and reasonably light $m_{1 / 2}$. However light selectrons and smuons also enhance $\delta a_{\mu}$ and, as a result, point S2 slightly exceeds the $2 \sigma$ bound on $\delta a_{\mu}{ }^{7}$.

Having access to $m_{0}=0$ also permits a solution to the SUSY flavour changing neutral current (FCNC) problem, at least for the first two generations, where it is most severe. Being zero at high energies, non-zero elements of the low energy upper block of the squark and slepton mass matrices proportional to the unit matrix are generated via gaugino running effects, giving a universal form involving the first two families, greatly suppressing the most severe FCNCs. This mechanism is analogous to "gaugino mediated SUSY breaking" [70, [1] but applies here to only the first two families. It was first studied in the framework of a particular brane set up in [72] where it was referred to as "brane mediated SUSY breaking".

Having considered the behaviour of a model with $m_{0,3}=1 \mathrm{TeV}$, we now go on to consider the case of general $m_{0,3}$ in Fig. 3. We fix $m_{0}=50 \mathrm{GeV}$ and allow $m_{0,3}$ to vary from $1-4 \mathrm{TeV}$. For the majority of the plot, the light shaded (green) region represents the region ruled out due to a smuon and selectron LSP. The dark matter strip that runs parallel to it is due to coannihilation with smuons and selectrons, as exemplified by point $\mathrm{S} 1$ in Fig. 3 also studied previously.

\footnotetext{
${ }^{6}$ Due to the definition of $\Delta^{\Omega}$, whenever $a=0, \Delta_{a}^{\Omega}=0$. However we have checked that this has not resulted in an artificially low $\Delta$ by taking a number of points of decreasing $m_{0}$. From this we conclude that as $m_{0} \rightarrow 0, \Delta_{m_{0}}^{\Omega} \rightarrow 0$ smoothly.

${ }^{7}$ As mentioned earlier this can be avoided if we allow a relative sign between $M_{1}$ and $M_{2}$.
} 


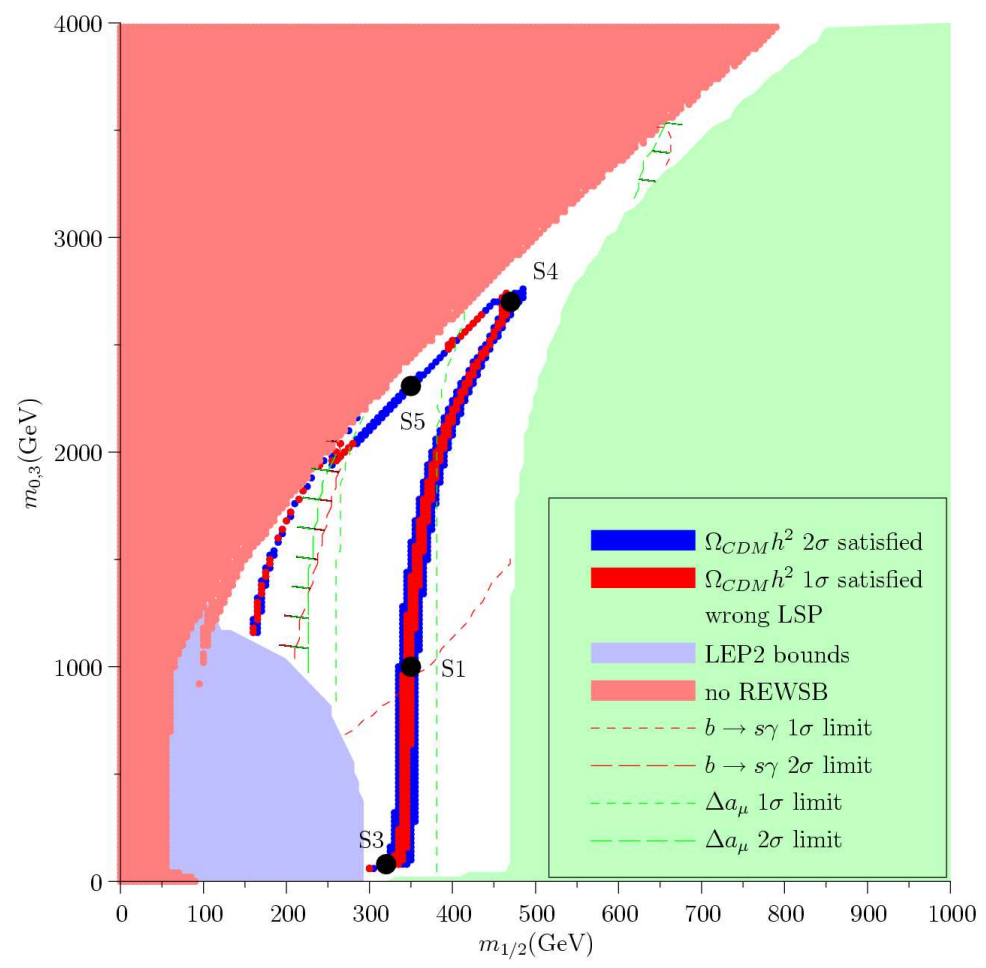

Figure 3: The $\left(m_{1 / 2}, m_{0,3}\right)$ plane for non-universal sfermion masses with $m_{0}=50 \mathrm{GeV}$, $A_{0}=0, \tan \beta=10$.

As we mentioned earlier, if we increase $m_{0,3}$ enough we lower $\mu^{2}$ to the point where it becomes negative and REWSB fails. As we approach the region in which REWSB fails, $|\mu|$ decreases and therefore we steadily increase the Higgsino component of the neutralino. This results in an analogue of the Focus Point region in the CMSSM. As well as an LSP with a significant Higgsino component we also have light charginos. These two factors together enhance decays of the form $\tilde{\chi}_{1}^{0} \tilde{\chi}_{1}^{0} \rightarrow W^{+} W^{-} \cdot \tilde{\chi}_{2}^{0}$ and $\tilde{\chi}_{3}^{0}$ also pick up substantial Higgsino components and thus become close in mass to $\tilde{\chi}_{1}^{0}$, allowing another form of coannihilation. All of these channels are most efficient when $\mu<M_{1}$ and the neutralino is pure Higgsino. In such cases the annihilation of SUSY matter in the early universe is so efficient that we would end up with no appreciable dark matter relic today. Therefore agreement with the observed relic density is achieved when the neutralino is a well tempered Bino/Higgsino mix, as in point S5 in Fig. 3.

The last band in Fig. 3 that agrees with $\Omega_{C D M} h^{2}$ is at low $m_{1 / 2}$ and $m_{0,3} \approx 1.2-$ $2 \mathrm{TeV}$. In this region $2 m_{\tilde{\chi}_{1}^{0}} \approx m_{h}$ and annihilation proceeds through resonant s-channel exchange of a light Higgs. However this region is ruled out at $2 \sigma$ by $B R(b \rightarrow s \gamma)$ and $\delta a_{\mu}$ so we do not study it further here.

To study the naturalness of the dark matter regions, we take the points S3-S5 as shown in Fig. 3 and Table 3. Point S1 also shown in Fig. 3 was considered previously. This allows a comparison of the Focus Point region with its well-tempered neutralino in point S5 to the smuon/selectron coannihilation region of point S1. We also take points S3 and S4 where these regions meet. Having found a hint of natural dark matter in 


\begin{tabular}{|l|l|l|l|}
\hline \multirow{2}{*}{ Parameter } & \multicolumn{3}{|l|}{ Value } \\
\cline { 2 - 4 } & S3 & S4 & S5 \\
\hline$\Delta_{m_{0}}^{\Omega}$ & 1.5 & 0.67 & 0.12 \\
$\Delta_{m_{0,3}}^{\Omega}$ & 0.41 & 2.4 & 30 \\
$\Delta_{m_{1 / 2}}^{\Omega}$ & 2.4 & 2.8 & 18 \\
$\Delta_{\tan \beta}^{\Omega}$ & 0.23 & 1.0 & 12 \\
$\Delta_{A_{0}}^{\Omega}$ & 0 & 0 & 0 \\
\hline$\Delta^{\Omega}$ & 2.4 & 2.8 & 30 \\
\hline$\Delta^{\mathrm{EW}}$ & 91 & 1300 & 950 \\
\hline
\end{tabular}

Table 3: Fine tuning sensitivity parameters at points in Fig. 3. S3 has $m_{0,3}=80 \mathrm{GeV}$, $m_{1 / 2}=320 \mathrm{GeV}$. S4 has $m_{0,3}=2700 \mathrm{GeV}, m_{1 / 2}=470 \mathrm{GeV}$. S5 has $m_{0,3}=2308 \mathrm{GeV}$, $m_{1 / 2}=350 \mathrm{GeV}$

point S2 in which we had more than one annihilation channel at work, S3 and S4 allow us to study the effect of regions that exhibit different combinations of annihilation channels.

Point S3 lies on the point at which selectrons, smuons and staus all contribute to annihilation rates equally. We also have a $47 \%$ contribution from t-channel slepton exchange. Because the stau coannihilation depends almost solely on $m_{0,3}$ and the selectron and smuon coannihilation depends on $m_{0}$, the sensitivity to either of these parameters is reduced. In addition, if we increase either $m_{0,3}$ or $m_{0}$ we are increasing the mass of either the staus or the smuons and selectrons, which always leaves at least one light slepton to mediate t-channel slepton exchange. As a result S3 has a lower fine tuning than either primarily stau coannihilation (as with $\mathrm{C} 1$ ) or selectron and smuon coannihilation (as with S1).

Point S4 also lies on an intersection in annihilation channels. This time it is the intersection of the Higgsino/Bino "well-tempered" strip and the selectron, smuon coannihilation strip. The quoted $\Delta_{a}^{\Omega}$ values should therefore be compared to S5 and S1 respectively. The selectron, smuon coannihilation strip is primarily sensitive to $m_{0}$ and $m_{1 / 2}$ through their effect on the sparticle masses. In contrast the Higgsino/Bino strip, represented by point S5, is highly sensitive to $m_{0,3}$ and $m_{1 / 2}$ through their effects on the low energy values of $\mu$ and $M_{1}$. Once again by combining channels we reduce our dependence on any one parameter. In this case we manage to achieve a low degree of fine-tuning while also having a well-tempered neutralino. However with $m_{0,3}=2700 \mathrm{GeV}$ we are in peril of reintroducing fine-tuning complaints in the Higgs sector.

Finally, note that the least natural region we have considered is that of the Higgsino/Bino neutralino without any other annihilation channels. In 34 the well-tempered neutralino is suggested as some of the most plausible options for a general SUSY theory. However here we have shown that, at least in certain models, such well-tempered regions are more fine-tuned with respect to soft parameters than coannihilation regions. 
In summary, allowing $m_{0,3}$ to vary independently of the first and second family masses, and in particular to become independently large, we find the following features:

- Access to $m_{0}=0$, and hence a solution to the SUSY FCNC problem, with lower $\Delta_{a}^{\Omega}$ than in the CMSSM at the expense of large SUSY contributions to $(g-2)_{\mu}$.

- Access to the well tempered Higgsino/Bino region which agrees with $\delta a_{\mu}$ at $1 \sigma$, but this well tempered point involves $3 \%$ dark matter fine tuning.

- We can access certain regions such as $\mathrm{S} 3, \mathrm{~S} 4$ in which we have a number of different annihilation channels at work and these lead to lower values of $\Delta_{a}^{\Omega}$ and more natural solutions to the dark matter problem.

- Large $m_{0,3}$ implies a high degree of fine tuning for REWSB, as expected, so a fully natural model is not possible in this case.

\section{Non-universal Gaugino Masses}

As with high 3rd family masses, there are good reasons for allowing the soft gaugino masses to be non-universal. From a theoretical point of view, non-universal gaugino masses are interesting as they naturally occur in SUGRA models with non-minimal gauge kinetic terms, which arise from many string constructions (see e.g. 69, 68]). They also naturally occur in gauge mediated SUSY breaking and anomaly mediated SUSY breaking, for example [73], [74] respectively.

From a phenomenological point of view, non-universal gauginos are extremely useful. By allowing $M_{1}$ and $M_{2}$ to take different values, we can directly change the Bino/Wino balance in the neutralinos allowing access to Wino and well tempered Bino/Wino LSP states. $M_{3}$ allows us to vary the squark sector independently of the sleptons through its effects on the running masses. $\mu$ also depends on $M_{3}$ as shown in Eq. 6. Therefore by allowing our gaugino masses to be non-universal we gain control over $M_{1}, M_{2}$ and to a lesser extent $\mu$.

To be precise we consider the parameters:

$$
m_{0}, M_{1}, M_{2}, M_{3}, \tan \beta, A_{0} \text { and } \operatorname{sign}(\mu)
$$

In Fig. 4 we keep $M_{2}=M_{3}=350 \mathrm{GeV}, \tan \beta=10$ and $A_{0}=0$, but allow $m_{0}$ and $M_{1}$ to vary. As we have a unified mass for the sfermions, the light shaded (green) region at low $m_{0}$ is excluded by a stau LSP. The LEP2 bound at $M_{1} \approx 100 \mathrm{GeV}$ is due to the neutralino becoming too light. With $M_{3}=350 \mathrm{GeV}$ the Higgs mass is above the LEP limit across the whole $\operatorname{scan}^{8}$.

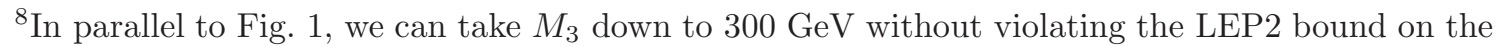
lightest Higgs. This also results in the lowest value of $\mu$.
} 


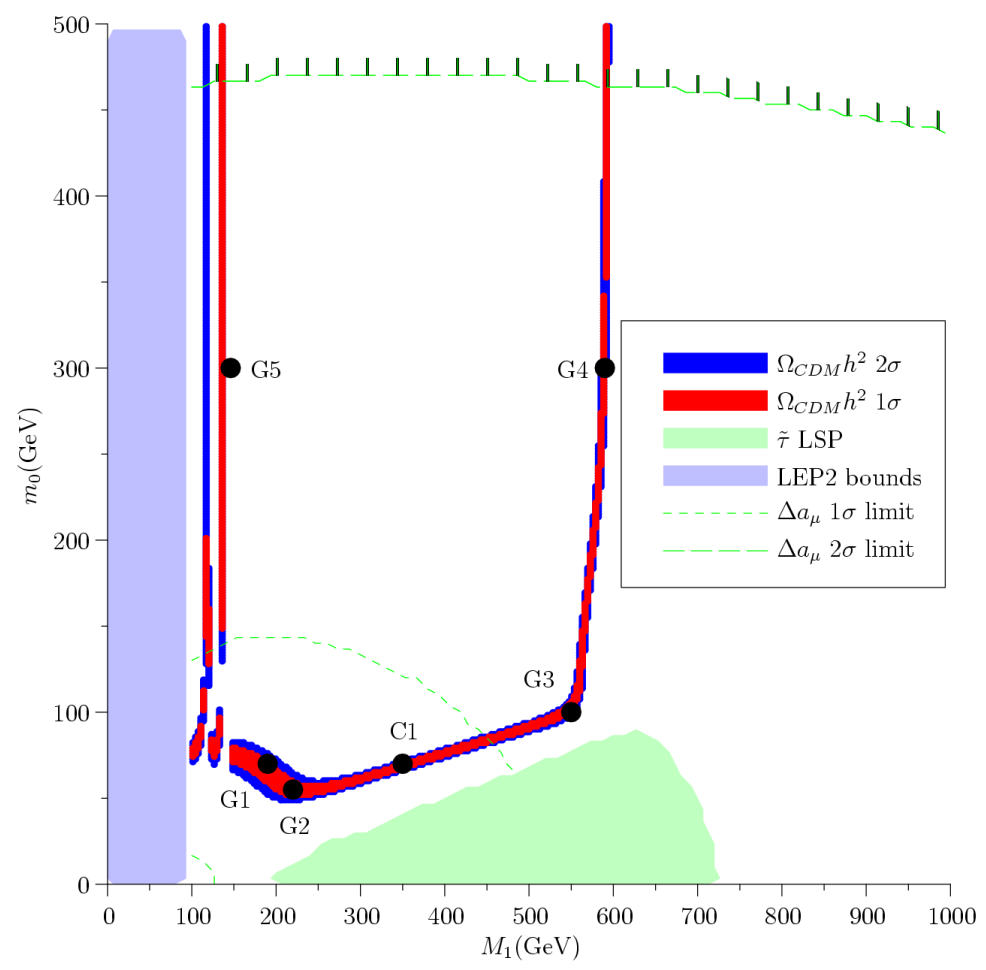

Figure 4: The $\left(M_{1}, m_{0}\right)$ plane for non-universal gauginos with $M_{2}=M_{3}=350 \mathrm{GeV}$, $A_{0}=0, \tan \beta=10$.

The most striking feature of Fig. 4 is the multitude of different regions that satisfy $\Omega_{C D M} h^{2}$. There is the coannihilation strip for $300 \mathrm{GeV}<M_{1}<500 \mathrm{GeV}$ containing the point $\mathrm{C} 1$ at $M_{1}=350 \mathrm{GeV}$ which coincides with the CMSSM point considered previously, with Bino dark matter and a stau coannihilation channel. In addition there is the well tempered Bino/Wino LSP at $M_{1} \approx 600 \mathrm{GeV}$ containing the point G4. This value of $M_{1}$ corresponds to the high energy relation $M_{1} \approx 1.7 M_{2}$ corresponding to the low energy equality $M_{1}\left(m_{Z}\right) \approx M_{2}\left(m_{Z}\right)$. The two vertical lines at $M_{1}=130 \mathrm{GeV}$ and $M_{1}=140 \mathrm{GeV}$ correspond to the points at which $2 m_{\tilde{\chi}_{1}^{0}}=m_{Z}, m_{h}$ respectively and we have resonant s-channel annihilation. However the most interesting region is the bulk region containing the point G1 which arises from the very low values of $150<M_{1}<250 \mathrm{GeV}$ which are allowed now that gaugino universality is relaxed, which allows the sleptons to be light enough that $\tilde{\chi}_{1}^{0} \tilde{\chi}_{1}^{0} \rightarrow l \bar{l}$ can produce the observed relic density on its own. We study fine tuning in these regions by calculating $\Delta_{a}^{\Omega}$ for points $\mathrm{C} 1, \mathrm{G} 4, \mathrm{G} 5, \mathrm{G} 1$ in each region and for points G2,G3 where two annihilation processes contribute equally. The results are presented in Table $4,{ }^{9}$

It is striking that two of the points in Table 4, namely G1 and G2, have a dark matter sensitivity parameter below unity, corresponding to "supernatural dark matter" (i.e. no fine tuning required at all to achieve successful dark matter). Point G1 lies

\footnotetext{
${ }^{9}$ Though point $\mathrm{C} 1$ has the same values for the soft parameters as in Table 1 here we allow the gaugino masses to vary independently. Therefore, though it is labelled $\mathrm{C} 1$, it does not represent the CMSSM. This is the point at which a model with non-universal gauginos makes contact with the CMSSM.
} 


\begin{tabular}{|l|l|l|l|l|l|l|}
\hline Parameter & \multicolumn{2}{|l|}{ Value } \\
\cline { 2 - 7 } & G1 & G2 & G3 & G4 & G5 & C1 \\
\hline$\Delta_{m_{0}}^{\Omega}$ & 0.83 & 0.97 & 3.0 & 0.65 & 5.7 & 3.5 \\
$\Delta_{M_{1}}^{\Omega}$ & 0.80 & 0.51 & 8.0 & 28 & 1100 & 2.7 \\
$\Delta_{M_{2}}^{\Omega}$ & 0.23 & 0.36 & 3.4 & 26 & 4.8 & 0.64 \\
$\Delta_{M_{3}}^{\Omega}$ & 0.24 & 0.44 & 2.3 & 5.8 & 91 & 1.4 \\
$\Delta_{\tan \beta}^{\Omega}$ & 0.20 & 0.50 & 1.2 & 0.20 & 4.1 & 1.4 \\
$\Delta_{A_{0}}^{\Omega}$ & 0 & 0 & 0 & 0 & 0 & 0 \\
\hline$\Delta^{\Omega}$ & 0.83 & 0.97 & 8.0 & 28 & 1100 & 3.5 \\
\hline$\Delta^{\mathrm{EW}}$ & 110 & 110 & 111 & 111 & 110 & 160 \\
\hline
\end{tabular}

Table 4: Fine tuning sensitivity parameters for points taken from Fig. 4 with $M_{2}=$ $M_{3}=350 \mathrm{GeV}, A_{0}=0, \tan \beta=10$. G1 has $m_{0}=70 \mathrm{GeV}, M_{1}=190 \mathrm{GeV} . \mathrm{G} 2$ has $m_{0}=55 \mathrm{GeV}, M_{1}=220 \mathrm{GeV}$. G3 has $m_{0}=100 \mathrm{GeV}, M_{1}=550 \mathrm{GeV}$ G4 has $m_{0}=300 \mathrm{GeV}, M_{1}=590 \mathrm{GeV}$ G5 has $m_{0}=300 \mathrm{GeV}, M_{1}=146.15 \mathrm{GeV}$.

in the middle of the bulk region in which annihilation proceeds through t-channel sfermion exchange. As this process depends directly upon the mass of the sleptons that are exchanged, it is unsurprising that the majority of the dependence is on $m_{0}$ and $M_{1}$. However it is clear that the dependence is significantly lower than the CMSSM. This is because the cross-section for $\tilde{\chi}_{1}^{0} \tilde{\chi}_{1}^{0} \rightarrow l \bar{l}$ varies slowly with $m_{\tilde{l}}$ in comparison to coannihilation processes that depend upon precise mass differences between particles. In the neighbouring point G2 both coannihilation and t-channel slepton exchange are competitive. Here the sensitivity to $m_{0}$ increases slightly (due to the coannihilation channel) but the sensitivity to $M_{1}$ decreases significantly for the same reasons as point $\mathrm{S} 2$ in the the case of non-universal scalars.

The advantage of combining annihilation channels is shown by comparing the point G3 and G4. G4 has been chosen to lie on the well-tempered Bino/Wino line. As with the Bino/Higgsino region, as we move away from a pure Bino LSP the annihilation and coannihilation cross-section rise dramatically, leading to a sharp drop in $\Omega_{C D M} h^{2}$. Here, as before, such "well-tempered" regions exhibit $\Delta_{a}^{\Omega}$ values of $\approx 30$, values well in excess of slepton coannihilation regions. G3 lies in the region in which we have both coannihilation with staus and a significant Wino component in the neutralino. This results in a region that is both "well-tempered" and exhibits lower values of $\Delta_{a}^{\Omega}$.

Finally we briefly consider the naturalness of s-channel annihilation regions. As these are sharply peaked whenever $2 m_{\tilde{\chi}_{1}^{0}}=m_{Z, h}$, we expect there to be a substantial dependence on any parameter that directly affect the neutralino mass. As point G5 shows, this dependence is extreme. If we were to find ourselves to be living in this part of parameter space we would have to look for some further theoretical justification. An $\Delta_{a}^{\Omega}$ value greater than 1000 cannot be considered natural.

Once again, these studies of naturalness can only be considered in the case of a given model. In this model with non-universal gaugino masses we have showed that the "welltempered" region is considerably more fine tuned than t-channel slepton exchange or even slepton coannihilation regions. However the tuning in the "well-tempered" region 
pales in significance when we consider the amount of tuning required to land us on the edge of the light Higgs annihilation channel.

However the fate of the Wino/Bino dark matter strip could be considered to be better than that of the Bino/Higgsino neutralino. The reason for this is that the Bino/Higgsino region depends precisely on $M_{1} / \mu$ whereas the Bino/Wino region depends on $M_{1} / M_{2}$. In the latter case to guarantee that we land in the Bino/Wino well-tempered strip it is only necessary to have a model that requires $M_{1}\left(m_{G U T}\right) \approx$ $1.7 M_{2}\left(m_{G U T}\right)$. In such a model the large dependence on $M_{1}$ and $M_{2}$ in G4 would disappear and a Wino/Bino neutralino could provide a dark matter candidate. However there is no magic ratio at the high scale that will obviously reproduce the relevant Higgsino/Bino ratio at the low scale. To see this it is enough to consider the dependence of $\mu$ on the soft parameters. To guarantee a given ratio between $\mu$ and $M_{1}$ it would be necessary to have a model that relates all the parameters in Eq. [6 in just the right way.

Leaving aside such questions of models of SUSY breaking, the most vivid result of allowing non-universal gaugino masses are:

- The bulk region involving Bino annihilation via t-channel slepton exchange can be accessed, since low $M_{1}$ is possible leading to light sleptons. This allows supernatural dark matter exemplified by the points G1,G2.

- Low $m_{0}$ and $M_{1}$ also allow us to fit $\delta a_{\mu}$ at $1 \sigma$.

- A well tempered Bino/Wino neutralino scenario is possible, for example point G4, but this requires $3 \%$ fine tuning as in the case of the well tempered Bino/Higgsino.

- However combinations of annihilation channels on the edge of such regions such as point G3 lead to lower fine tuning.

- To achieve the observed value of $\Omega_{C D M} h^{2}$ through resonant annihilation such as at point G5 through a light Higgs requires extremely large fine tuning.

\section{Non-universal Gauginos and Third Family Scalar Masses}

Having considered non-universal gauginos and the situation in which we allow the third family sfermion and Higgs masses to be large, we now consider the effect of including both of these extensions to the CMSSM at once. This results in a model with 7 free soft parameters and a sign:

$$
m_{0}, m_{0,3}, M_{1}, M_{2}, M_{3}, \tan \beta, A_{0} \text { and } \operatorname{sign}(\mu)
$$

It is clear from the two previous sections that the mechanics of dark matter annihilation can be sensitive to many of these parameters. To get a true handle of the 


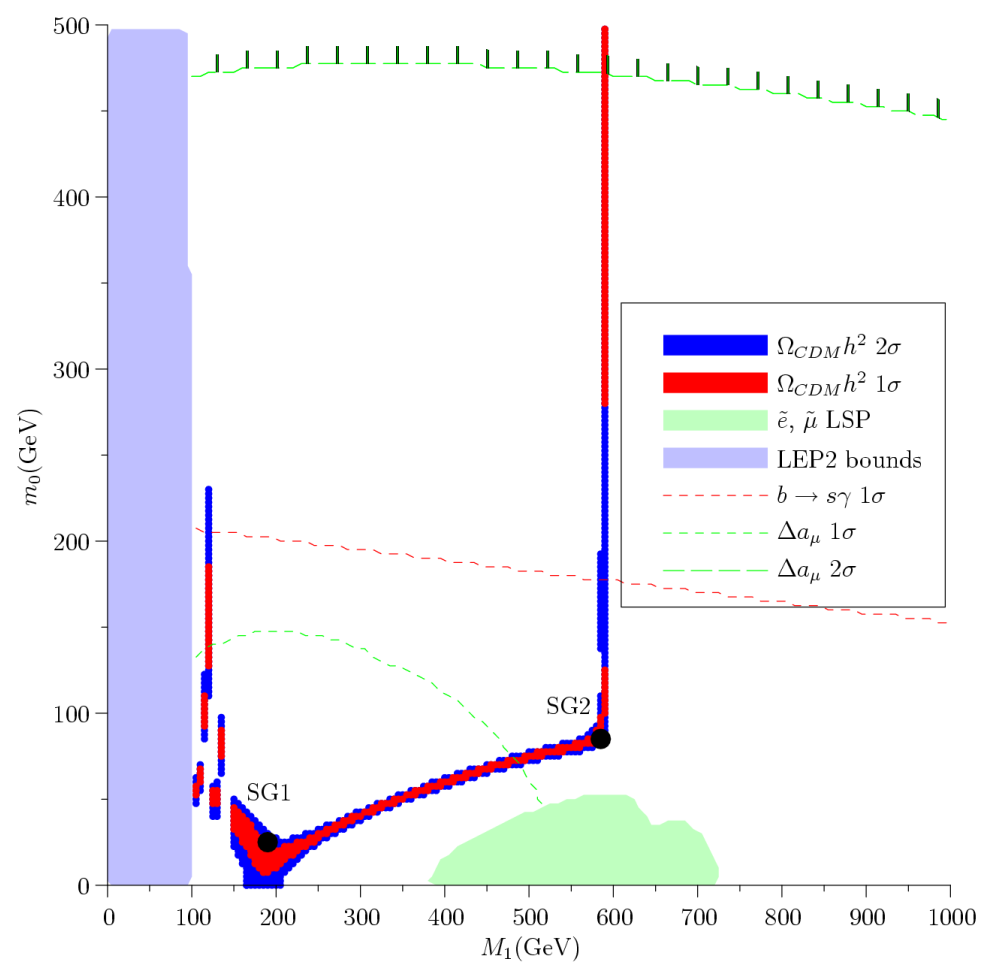

Figure 5: The $\left(M_{1}, m_{0}\right)$ plane for non-universal gaugino and sfermion masses with $M_{2}=M_{3}=350 \mathrm{GeV}, m_{0,3}=1000 \mathrm{GeV}, A_{0}=0, \tan \beta=10$.

sensitivity of these regions to the soft parameters, we should allow all of the different parameters to vary at once. In addition, by allowing all parameters to vary simultaneously, we open up the possibility of accessing new regions in which we satisfy dark matter that have even lower $\Delta_{a}^{\Omega}$ values than we have found so far. However having large $m_{0,3}$ clearly means that REWSB will be fine tuned. Nevertheless a new result of having both types of non-universality together is that we can access a maximally tempered Bino/Wino/Higgsino neutralino where the LSP consists of roughly equal amounts of Bino, Wino and Higgsino.

In Fig. 5 we take $M_{2,3}=350 \mathrm{GeV}, \tan \beta=10$ and $A_{0}=0$ as in 4 but set $m_{0,3}=1 \mathrm{TeV}$. The introduction of a high 3rd family mass has had the same general effects as in Fig. 2. The light shaded (green) excluded region is now due to smuon and selectron LSPs rather than staus which once again allows access to $m_{0}=0$. The lack of a light stau has also reduced the $\tilde{\chi}_{1}^{0} \tilde{\chi}_{1}^{0} \rightarrow l \bar{l}$ cross-section across the entire parameter space. Thus we need lower values of $m_{0}$ and $M_{1}$, and thus lighter smuons and selectrons, to access a region in which t-channel slepton exchange alone can satisfy dark matter constraints.

Apart from these details, the general features remain the same as in Fig. 田 We cannot access $M_{1}<100 \mathrm{GeV}$ due to LEP2 bounds on the lightest neutralino. At low $M_{1}$ we have thin lines corresponding to the light Higgs and $\mathrm{Z}$ resonances. The line is broken due to the resolution of the grid used to scan the space - a testament to extreme sensitivity of these regions to $M_{1}$. At low $M_{1}$ and low $m_{0}$ we have dominant 
contributions from t-channel slepton exchange. For moderate values of $M_{1}$ we have coannihilation with selectrons and smuons and when $M_{1} \approx 600 \mathrm{GeV}$, we once again get Bino/Wino dark matter.

\begin{tabular}{|l|l|l|}
\hline \multirow{2}{*}{ Parameter } & \multicolumn{2}{|c|}{ Value } \\
\cline { 2 - 3 } & SG1 & SG2 \\
\hline$\Delta_{m_{0}}^{\Omega}$ & 0.25 & 1.6 \\
$\Delta_{m_{0,3}}^{\Omega}$ & 0.073 & 0.15 \\
$\Delta_{M_{1}}^{\Omega}$ & 0.62 & 8.9 \\
$\Delta_{M_{2}}^{\Omega}$ & 0.0080 & 7.7 \\
$\Delta_{M_{3}}^{\Omega}$ & 0.027 & 0.89 \\
$\Delta_{\text {tan } \beta}^{\Omega}$ & 0.0020 & 0.056 \\
$\Delta_{A_{0}}^{\Omega}$ & 0 & 0 \\
\hline$\Delta^{\Omega}$ & 0.62 & 8.9 \\
\hline$\Delta^{\mathrm{EW}}$ & 240 & 240 \\
\hline
\end{tabular}

Table 5: Fine tuning sensitivity at points taken from Fig. 5 with $M_{2}=M_{3}=350 \mathrm{GeV}$, $m_{0,3}=1000 \mathrm{GeV}, A_{0}=0, \tan \beta=10$. SG1 has $m_{0}=25 \mathrm{GeV}, M_{1}=190 \mathrm{GeV}$. SG2 has $m_{0}=85 \mathrm{GeV}, M_{1}=585 \mathrm{GeV}$.

In our hunt for natural dark matter, we once again consider points in which many different annihilation channels contribute. We take point SG1 at the intersection of the selectron, smuon coannihilation region and the t-channel slepton band. Point SG2 is taken in the region where Bino/Wino dark matter also co-annihilates with selectrons and smuons. SG1 once again shows that regions with low $m_{0}$ and $M_{1}$ exhibit small fine-tuning. Here the dark matter band appears at lower $m_{0}$ and lower $M_{1}$ in Fig. [5 than in Fig. 2. The result, through a combination of the effects discussed earlier, is an order of magnitude decrease in the sensitivity of this region in comparison to the most favourable region of the CMSSM. Point SG1 again provides a supernatural solution to dark matter ${ }^{10}$.

Fig. [6]contains the maximally tempered neutralino at point SG3. With $M_{2}=M_{3}=$ $350 \mathrm{GeV}$, we take $m_{0,3}=2250 \mathrm{GeV}$ to achieve small enough $\mu$. Once again this raises questions of fine-tuning in the electroweak sector but we leave such concerns aside for the time being. The region of interest lies at $M_{1} \approx 400 \mathrm{GeV}$. This line is rather disjointed as SOFTSUSY has difficulty calculating the spectrum in regions where $\mu$ is small. In this band the neutralino has significant portions of Bino, Wino and Higgsino. This results in all of the neutralinos and charginos being close in mass and so results in a large number of annihilation and coannihilation processes in the early universe. In Table 6 we study point SG3 at $m_{0}=0 \mathrm{GeV}, M_{1}=375 \mathrm{GeV}$.

The "maximally tempered" neutralino at point SG3 exhibits dramatically lower fine-tuning than either the Bino/Wino (G4) or Bino/Higgsino (S5) regions. By allowing the neutralino to be maximally mixed, we decrease the degree of tuning required to

\footnotetext{
${ }^{10}$ In this region we also agree with $\delta a_{\mu}$ in contrast to $m_{0}=0$ point in section 5 This is because $M_{2}=350 \mathrm{GeV}$ which avoids charginos that are too light and keeps $\delta a_{\mu}$ at the correct level.
} 


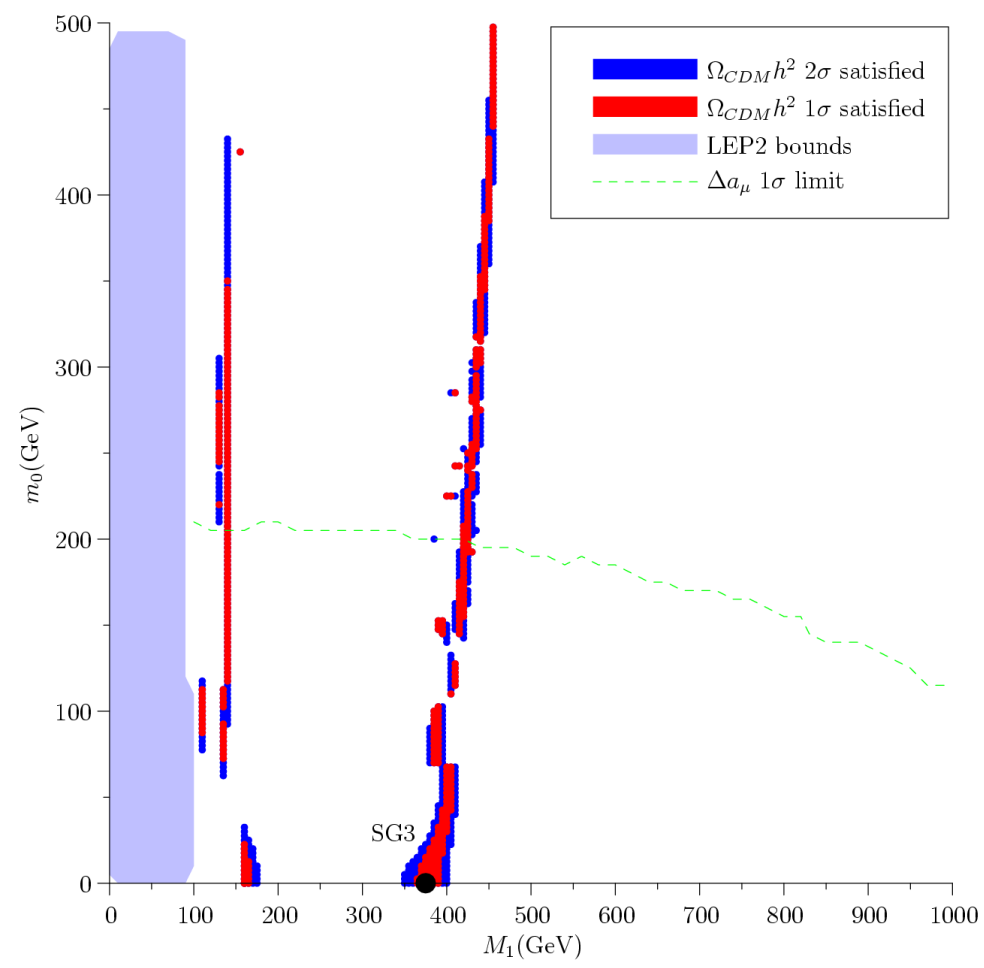

Figure 6: The $\left(M_{1}, m_{0}\right)$ plane for non-universal gaugino and sfermion masses with $M_{2}=M_{3}=350 \mathrm{GeV}, m_{0,3}=2250 \mathrm{GeV}, A_{0}=0, \tan \beta=10$.

satisfy dark matter by an order of magnitude with respect to merely "well-tempered" cases.

As with the case of non-universal gaugino masses alone, or just a high third family scalar mass, we find that there are certain sweet points in parameter spaces where different annihilation channels contribute with roughly equal strength. These regions are characterised by a sharp drop in the fine-tuning of soft parameters required to reproduce $\Omega_{C D M} h^{2}$. By allowing both non-universal soft gaugino masses and nonuniversal 3rd family scalar masses we find that not only are these sweet spots stable against further non-universalities, but also that we can access values of $\Delta_{a}^{\Omega}$ an order of magnitude smaller than in the CMSSM.

\section{Conclusions}

We have explored regions of MSSM parameter space with non-universal gaugino and third family scalar masses in which neutralino dark matter may be implemented naturally. In order to examine the relative naturalness of different regions we employed a dark matter fine-tuning sensitivity parameter, which we use in conjunction with the similarly defined sensitivity parameter used for EWSB. Employing these quantitative measures of fine-tuning we find that $\tilde{\tau}$-coannihilation channel in the CMSSM may involve as little as $25 \%$ tuning, due to renormalisation group ( $R G$ ) running effects. 


\begin{tabular}{|l|l|}
\hline Parameter & Value \\
\cline { 2 - 2 } & SG3 \\
\hline$\Delta_{m_{0}}^{\Omega}$ & 0.064 \\
$\Delta_{m_{0,3}}^{\Omega}$ & 3.8 \\
$\Delta_{M_{1}}^{\Omega}$ & 2.0 \\
$\Delta_{M_{2}}^{\Omega}$ & 0.52 \\
$\Delta_{M_{3}}^{\Omega}$ & 3.7 \\
$\Delta_{\tan \beta}^{\Omega}$ & 1.0 \\
$\Delta_{A_{0}}^{\Omega}$ & 0.015 \\
\hline$\Delta^{\Omega}$ & 3.8 \\
\hline$\Delta^{\mathrm{EW}}$ & 240 \\
\hline
\end{tabular}

Table 6: Fine tuning sensitivity at the maximally tempered point SG3 taken from [6] with $M_{2}=M_{3}=350 \mathrm{GeV}, m_{0,3}=1000 \mathrm{GeV}, A_{0}=0, \tan \beta=10$. SG3 has $m_{0}=0 \mathrm{GeV}, M_{1}=375 \mathrm{GeV}$.

For non-universal third family scalar masses in which $m_{0,3}$ is allowed to become independently large, we find that we have access to $m_{0}=0$, and hence a solution to the SUSY FCNC problem, with lower $\Delta_{a}^{\Omega}$ than in the CMSSM at the expense of large SUSY contributions to $(g-2)_{\mu}$. Alternatively, with non-universal third family scalar masses, we can also access the well tempered Higgsino/Bino region which agrees with $\delta a_{\mu}$ at $1 \sigma$, but this well tempered point involves $3 \%$ dark matter fine tuning. We can access certain regions in which we have a number of different annihilation channels at work and these lead to lower values of dark matter fine tuning. However large $m_{0,3}$ implies a high degree of fine tuning for REWSB, as expected, so a fully natural model is not possible in this case.

With non-universal gauginos the bulk region involving Bino annihilation via tchannel slepton exchange can be accessed, since low $M_{1}$ is possible leading to light sleptons. This allows supernatural dark matter (for example at points G1, G2) with minimal EWSB fine tuning, depending on how low $M_{3}$ is taken consistently with the LEP Higgs bound. Low $m_{0}$ and $M_{1}$ also allow us to fit $\delta a_{\mu}$ at $1 \sigma$. Alternatively, with non-universal gauginos, the well tempered Bino/Wino neutralino scenario is also possible, but again requires 3\% fine tuning as in the case of the well tempered Bino/Higgsino. However combinations of annihilation channels on the edge of such regions such as point G3 lead to lower fine tuning. To achieve the observed value of $\Omega_{C D M} h^{2}$ through resonant annihilation such as at point G5 through a light Higgs requires extremely large fine tuning.

With both non-universal third family masses and non-universal gauginos, a new feature appears: the "maximally tempered" Bino/Wino/Higgsino neutralino where the LSP consists of roughly equal amounts of Bino, Wino and Higgsino (for example point SG3). Although the maximally tempered neutralino has quite low fine tuning, the large value of $m_{0,3}$ inherent in this approach always means that EWSB will be very fine tuned.

In general, having non-universal third family and gaugino masses opens up new 
regions of MSSM parameter space in which dark matter may be implemented naturally. In particular allowing non-universal gauginos opens up the bulk region that allows Bino annihilation via t-channel slepton exchange, leading to "supernatural dark matter" corresponding to no fine-tuning at all with respect to dark matter. By contrast we find that the recently proposed "well tempered neutralino" regions involve substantial finetuning of MSSM parameters in order to satisfy the dark matter constraints, although the fine tuning may be ameliorated if several annihilation channels act simultaneously. Although we have identified regions of "supernatural dark matter" in which there is no fine tuning to achieve successful dark matter, the usual MSSM fine tuning to achieve EWSB always remains.

\section{Acknowledgements}

SFK would like to thank John Ellis and the members of the CERN Cosmo Coffee Club for helpful comments. SFK would also like to thank Lee Roberts for discussing the latest results relevant to the muon $g-2$ discrepancy. JPR acknowledges a PPARC Studentship.

\section{References}

[1] D. J. H. Chung, L. L. Everett, G. L. Kane, S. F. King, J. D. Lykken and L. T. Wang, Phys. Rept. 407 (2005) 1 arXiv:hep-ph/0312378.

[2] G. Jungman, M. Kamionkowski and K. Griest, Phys. Rept. 267 (1996) 195 arXiv:hep-ph/9506380.

[3] J. R. Ellis, S. Heinemeyer, K. A. Olive and G. Weiglein, JHEP 0502 (2005) 013 arXiv:hep-ph/0411216.

[4] G. Belanger, F. Boudjema, A. Cottrant, A. Pukhov and A. Semenov, Nucl. Phys. B 706 (2005) 411 arXiv:hep-ph/0407218.

[5] H. Baer, T. Krupovnickas, A. Mustafayev, E. K. Park, S. Profumo and X. Tata, arXiv:hep-ph/0511034.

[6] H. Baer, A. Mustafayev, E. K. Park and S. Profumo, JHEP 0507, 046 (2005) arXiv:hep-ph/0505227.

[7] S. Baek, D. G. Cerdeno, Y. G. Kim, P. Ko and C. Munoz, JHEP 0506, 017 (2005) arXiv:hep-ph/0505019.

[8] D. G. Cerdeno and C. Munoz, JHEP 0410, 015 (2004) arXiv:hep-ph/0405057.

[9] E. Nezri, arXiv:hep-ph/0305114.

[10] A. Birkedal-Hansen and B. D. Nelson, Phys. Rev. D 64, 015008 (2001) arXiv:hep-ph/0102075. 
[11] H. Baer, A. Mustafayev, E. K. Park, S. Profumo and X. Tata, JHEP 0604, 041 (2006) arXiv:hep-ph/0603197.

[12] K. Huitu, J. Laamanen, P. N. Pandita and S. Roy, Phys. Rev. D 72, 055013 (2005) arXiv:hep-ph/0502100.

[13] C. Pallis, Nucl. Phys. B 678, 398 (2004) arXiv:hep-ph/0304047.

[14] A. Corsetti and P. Nath, Phys. Rev. D 64, 125010 (2001) arXiv:hep-ph/0003186.

[15] U. Chattopadhyay and P. Nath, Phys. Rev. D 65, 075009 (2002) arXiv:hep-ph/0110341.

[16] U. Chattopadhyay, A. Corsetti and P. Nath, Phys. Rev. D 66, 035003 (2002) arXiv:hep-ph/0201001.

[17] H. Baer, A. Belyaev, T. Krupovnickas and A. Mustafayev, JHEP 0406 (2004) 044 arXiv:hep-ph/0403214.

[18] M. R. Ramage and G. G. Ross, JHEP 0508 (2005) 031 arXiv:hep-ph/0307389.

[19] E. Accomando, R. Arnowitt and B. Dutta, Phys. Lett. B 475, 176 (2000) arXiv:hep-ph/9811300.

[20] D. Auto, H. Baer, A. Belyaev and T. Krupovnickas, JHEP 0410, 066 (2004) arXiv:hep-ph/0407165.

[21] M. Olechowski and S. Pokorski, Phys. Lett. B 344 (1995) 201 arXiv:hep-ph/9407404.

[22] F. M. Borzumati, M. Olechowski and S. Pokorski, Phys. Lett. B 349 (1995) 311 arXiv:hep-ph/9412379.

[23] V. Berezinsky, A. Bottino, J. R. Ellis, N. Fornengo, G. Mignola and S. Scopel, Astropart. Phys. 5 (1996) 1 arXiv:hep-ph/9508249.

[24] P. Nath and R. Arnowitt, Phys. Rev. D 56 (1997) 2820 arXiv:hep-ph/9701301.

[25] E. Accomando, R. Arnowitt, B. Dutta and Y. Santoso, Nucl. Phys. B 585 (2000) 124 arXiv:hep-ph/0001019.

[26] R. Arnowitt and B. Dutta, arXiv:hep-ph/0210339.

[27] R. Dermisek, S. Raby, L. Roszkowski and R. Ruiz De Austri, JHEP 0304 (2003) 037 arXiv:hep-ph/0304101.

[28] V. Bertin, E. Nezri and J. Orloff, JHEP 0302 (2003) 046 arXiv:hep-ph/0210034.

[29] A. Birkedal-Hansen and B. D. Nelson, Phys. Rev. D 67 (2003) 095006 arXiv:hep-ph/0211071.

[30] U. Chattopadhyay and D. P. Roy, Phys. Rev. D 68 (2003) 033010 arXiv:hep-ph/0304108. 
[31] D. G. Cerdeno, K. Y. Choi, K. Jedamzik, L. Roszkowski and R. Ruiz de Austri, arXiv:hep-ph/0509275.

[32] L. Covi, L. Roszkowski, R. Ruiz de Austri and M. Small, JHEP 0406 (2004) 003 arXiv:hep-ph/0402240.

[33] G. W. Bennett et al. [Muon g-2 Collaboration], Phys. Rev. Lett. 92 (2004) 161802 arXiv:hep-ex/0401008.

[34] N. Arkani-Hamed, A. Delgado and G. F. Giudice, arXiv:hep-ph/0601041.

[35] R. Barbieri and G. F. Giudice, Nucl. Phys. B 306 (1988) 63.

[36] G. G. Ross and R. G. Roberts, Nucl. Phys. B 377 (1992) 571.

[37] B. de Carlos and J. A. Casas, Phys. Lett. B 309, 320 (1993) arXiv:hep-ph/9303291.

[38] S. Dimopoulos and G. F. Giudice, Phys. Lett. B 357, 573 (1995) arXiv:hep-ph/9507282.

[39] P. H. Chankowski, J. R. Ellis and S. Pokorski, Phys. Lett. B 423, 327 (1998) arXiv:hep-ph/9712234.

[40] R. Barbieri and A. Strumia, Phys. Lett. B 433, 63 (1998) arXiv:hep-ph/9801353.

[41] P. H. Chankowski, J. R. Ellis, M. Olechowski and S. Pokorski, Nucl. Phys. B 544 (1999) 39 arXiv:hep-ph/9808275.

[42] G. L. Kane and S. F. King, Phys. Lett. B 451 (1999) 113 arXiv:hep-ph/9810374.

[43] J. L. Feng, K. T. Matchev and T. Moroi, Phys. Rev. Lett. 84, 2322 (2000) arXiv:hep-ph/9908309.

[44] J. L. Feng, K. T. Matchev and T. Moroi, Phys. Rev. D 61 (2000) 075005 arXiv:hep-ph/9909334.

[45] M. Bastero-Gil, G. L. Kane and S. F. King, Phys. Lett. B 474, 103 (2000) arXiv:hep-ph/9910506.

[46] A. Romanino and A. Strumia, Phys. Lett. B 487, 165 (2000) arXiv:hep-ph/9912301.

[47] J. A. Casas, J. R. Espinosa and I. Hidalgo, JHEP 0401, 008 (2004) arXiv:hep-ph/0310137.

[48] B. C. Allanach and C. G. Lester, Phys. Rev. D 73, 015013 (2006) arXiv:hep-ph/0507283.

[49] B. C. Allanach, Phys. Lett. B 635, 123 (2006) arXiv:hep-ph/0601089.

[50] J. R. Ellis and K. A. Olive, Phys. Lett. B 514 (2001) 114 arXiv:hep-ph/0105004. 
[51] J. R. Ellis, K. A. Olive and Y. Santoso, New J. Phys. 4 (2002) 32 arXiv:hep-ph/0202110.

[52] G. W. Anderson and D. J. Castano, Phys. Rev. D 53, 2403 (1996) arXiv:hep-ph/9509212.

[53] G. W. Anderson and D. J. Castano, Phys. Rev. D 52, 1693 (1995) arXiv:hep-ph/9412322.

[54] G. W. Anderson and D. J. Castano, Phys. Lett. B 347, 300 (1995) arXiv:hep-ph/9409419.

[55] B. C. Allanach, Comput. Phys. Commun. 143 (2002) 305 arXiv:hep-ph/0104145.

[56] G. Belanger, F. Boudjema, A. Pukhov and A. Semenov, Comput. Phys. Commun. 149 (2002) 103 arXiv:hep-ph/0112278.

[57] K. Abe et al. [Belle Collaboration], Phys. Lett. B 511 (2001) 151 arXiv:hep-ex/0103042.

[58] D. Cronin-Hennessy et al. [CLEO Collaboration], Phys. Rev. Lett. 87 (2001) 251808 arXiv:hep-ex/0108033.

[59] R. Barate et al. [ALEPH Collaboration], Phys. Lett. B 429 (1998) 169.

[60] J. R. Ellis, Int. J. Mod. Phys. A 20 (2005) 5297 arXiv:hep-ph/0409360.

[61] A. Hocker, arXiv:hep-ph/0410081.

[62] See talk by M. N. Achasov, at the International Workshop on $e^{+} e^{-}$Collisions from $\phi$ to $\Psi$, February 27 - March 2, 2006, BINP, Novosibirsk, Russia (web page http://www.inp.nsk.su/conf/phipsi06/).

[63] S. P. Martin and J. D. Wells, Phys. Rev. D 64 (2001) 035003 arXiv:hep-ph/0103067.

[64] D. N. Spergel et al. [WMAP Collaboration], Astrophys. J. Suppl. 148 (2003) 175 arXiv:astro-ph/0302209.

[65] J. L. Feng, K. T. Matchev and F. Wilczek, Phys. Lett. B 482 (2000) 388 arXiv:hep-ph/0004043.

[66] K. Griest and D. Seckel, Phys. Rev. D 43 (1991) 3191.

[67] J. R. Ellis, T. Falk, K. A. Olive and M. Srednicki, Astropart. Phys. 13 (2000) 181 [Erratum-ibid. 15 (2001) 413] arXiv:hep-ph/9905481.

[68] S. F. King and D. A. J. Rayner, arXiv:hep-ph/0211242.

[69] B. C. Allanach, S. F. King and D. A. J. Rayner, JHEP 0405 (2004) 067 arXiv:hep-ph/0403255.

[70] H. Baer, C. Balazs, A. Belyaev, R. Dermisek, A. Mafi and A. Mustafayev, JHEP 0205 (2002) 061 arXiv:hep-ph/0204108. 
[71] Z. Chacko, M. A. Luty, A. E. Nelson and E. Ponton, JHEP 0001 (2000) 003 arXiv:hep-ph/9911323.

[72] S. F. King and D. A. J. Rayner, Nucl. Phys. B 607 (2001) 77 arXiv:hep-ph/0012076.

[73] S. Dimopoulos, G. F. Giudice and A. Pomarol, Phys. Lett. B 389 (1996) 37 arXiv:hep-ph/9607225.

[74] J. L. Feng and T. Moroi, Phys. Rev. D 61 (2000) 095004 arXiv:hep-ph/9907319. 